\title{
Population and mutation analysis of Y-STR loci in a sample from the city of São Paulo (Brazil)
}

\author{
José A. Soares-Vieira ${ }^{1}$, Ana E.C. Billerbeck ${ }^{2}$, Edna S.M. Iwamura ${ }^{1}$, Berenice B. Mendonca ${ }^{2}$, \\ Leonor Gusmão ${ }^{3}$ and Paulo A. Otto ${ }^{4}$ \\ ${ }^{1}$ Departamento de Medicina Legal, Faculdade de Medicina, Universidade de São Paulo, São Paulo, \\ SP, Brazil. \\ ${ }^{2}$ Laboratório de Hormônios e Genética Molecular, $1^{a}$ Clínica Médica, Hospital das Clínicas, \\ Faculdade de Medicina, Universidade de São Paulo, São Paulo, SP, Brazil. \\ ${ }^{3}$ Instituto de Patologia e Imunologia Molecular, Universidade do Porto, Porto, Portugal. \\ ${ }^{4}$ Departamento de Genética e Biologia Evolutiva, Instituto de Biociências, Universidade de São Paulo, \\ São Paulo, SP, Brazil.
}

\begin{abstract}
The haplotypes of seven Y-chromosome STR loci (DYS19, DYS389I, DYS389II, DYS390, DYS391, DYS392, and DYS393) were determined in a sample of 634 healthy Brazilian males (190 adult individuals and 222 father-son pairs). The 412 adults were unrelated, and the 222 father-son pairs had their biological relationship confirmed using autosomal STRs (LR > 10,000). Among the 412 adults, a total of 264 different 7-loci haplotypes were identified, 210 of which were unique. The most frequent haplotype was detected in 31 instances, occurring with a frequency of $7.52 \%$. The haplotype diversity index was calculated as $98.83 \%$. Upon transmission of the 1,554 alleles, in 222 father-son pairs, six mutations were observed, with an average overall rate of $3.86 \times 10^{-3}$ per locus. A haplotype with a duplicated DYS389I locus, and another with duplicated DYS389I, DYS389II, and DYS439 loci were detected in both fathers and their respective sons.
\end{abstract}

Key words: Y-STR population data, São Paulo (Brazil), mutation rates, duplications.

Received: March 14, 2008; Accepted: June 9, 2008.

Y-chromosome STR typing has become an important tool in forensic analysis (Betz et al., 2001; Sibille et al., 2002; Cerri et al., 2003; Shewale et al., 2003; Shewale et al., 2004; Delfin et al., 2005; Johnson et al., 2005). Recently, the DNA Commission of the International Society of Forensic Genetics (ISFG) has published guidelines and recomendations concerning the use of Y-STRs polymorphisms in human identification and kinship analysis (Gusmão et al., 2005). According to a recent Brazilian government census (IBGE), $54 \%$ of Brazilians were self-declared as white, $38 \%$ as mixed (mulatto), and $6 \%$ as black; $2 \%$ were classified in other categories that include Orientals and Amerindians, with striking regional differences. For instance, mixed tri-hybrid types are overwhelmingly predominant (almost 100\%) in some parts of the northeastern region, whereas whites vastly predominate in the southern

Send correpondence to José Arnaldo Soares-Vieira. Departamento de Medicina Legal, Faculdade de Medicina, Universidade de São Paulo, Rua Teodoro Sampaio 115, 05405-000, São Paulo, SP, Brazil. E-mail: jasv@usp.br. states (almost 100\% in some inner regions of the states of Santa Catarina and Rio Grande do Sul). Several studies performed in different population samples from Brazil (Costa et al., 2002; Grattapaglia et al., 2005; Cainé et al., 2005;; Carvalho-Silva et al., 2006; Silva et al., 2006; Domingues et al., 2007; Palha et al., 2007) have shown, however, that in spite of this racial melting pot, genes carried on the $\mathrm{Y}$ chromosome are almost exclusively of European origin (Iberian, Mediterranean, and Central-European), while analyses of mtDNA variability in Brazilian samples revealed that about $60 \%$ of the maternal lineages are Amerindian and African (Carvalho-Silva et al., 2006). Therefore, regardless of this intense gene flow and high degree of genetic heterogeneity, Y chromosome polymorphisms in Brazilian males have a distribution typical of a mixed European population. This paper presents data on 7 Y-STR loci DYS19, DYS389I, DYS389II, DYS390, DYS391, DYS392, and DYS393 in a Brazilian mixed population sample from the city of São Paulo. 
Whole blood samples were collected from 634 healthy Brazilian males (190 adult individuals and 222 pairs of fathers and respective sons), under written informed consent. The 412 adults were unrelated and the 222 father-son pairs had their biological relationship confirmed by paternity index values larger than 10,000 obtained by means of autosomal STRs typing. DNA was extracted from $5 \mathrm{~mL}$ of peripheral blood by a salting-out procedure (Miller et al., 1988), and quantified by spectrometry (Ultrospec III, Pharmacia, Piscataway, NJ, USA). The amplification of DYS19, DYS389I, DYS389II, DYS390, DYS391, DYS392 and DYS393 loci was performed according to Kayser et al. (1997), in two multiplex reactions, one triplex (DYS391,DYS392, DYS393), and one tetraplex (DYS19, DYS389I, DYS389II and DYS390). One primer of each pair was labeled with a fluorescent dye. In a final volume of $25 \mu \mathrm{L}, 50 \mathrm{ng}$ of genomic DNA was mixed with $200 \mu \mathrm{M}$ of dNTP, $2.0 \mathrm{mM} \mathrm{MgCl} 2,2.5 \mathrm{U}$ of Taq polymerase (Amersham Biosciences, Piscataway, NJ, USA), $2.5 \mu \mathrm{L}$ of the $10 \mathrm{X}$ reaction buffer provided by the manufacturer, and with the forward and reverse primers. In the triplex reaction, the concentrations of primers were 7.0 pmol for DYS391, 8.5 pmol for DYS392 and 3.0 pmol for DYS393; in the tetraplex reaction, $7.0 \mathrm{pmol}$ for DYS19, $6.0 \mathrm{pmol}$ for DYS389I/II and 4.0 pmol for DYS390. The samples were subjected to 30 cycles of amplification in a 9700 thermal cycler (Applied Biosystems, Foster City, CA). The amplification conditions were $94{ }^{\circ} \mathrm{C}, 5 \mathrm{~min} ; 35$ cycles of $94{ }^{\circ} \mathrm{C}$ $1 \mathrm{~min}, 55^{\circ} \mathrm{C} 1 \mathrm{~min}$, and $72{ }^{\circ} \mathrm{C} 1 \mathrm{~min}$; followed by $72{ }^{\circ} \mathrm{C}$ $30 \mathrm{~min}$, and $12^{\circ} \mathrm{C}$ until the samples were removed from the thermal cycler. Fragment size analysis was performed using the GeneScan 2.1 software. Two microliters of the amplification products were mixed with $24 \mu \mathrm{L}$ of $\mathrm{Hi}-\mathrm{Di}$ Formamide (Applied Biosystems), $1 \mu \mathrm{L}$ of the size standard TAMRA-350 (triplex reaction) or TAMRA-500 (tetraplex reaction) and subjected to capillary electrophoresis on the ABI 310 Genetic Analyzer (Applied Biosystems) using POP-4 (performance optimized polymer), filter set $\mathrm{C}$ and an injection time of $5 \mathrm{~s}$. The electrophoresis time was $24 \mathrm{~min}$ for the triplex reaction and $28 \mathrm{~min}$ for the tetraplex reaction. Four samples were reanalyzed using the AmpFlSTR YFiler kit (Applied Biosystems) as recommended by the manufacturer. Since Y-STRs are haploid, allele and haplotype frequencies, as well as linkage disequilibrium values between pairs of genes at different loci and mutation rates per locus, were estimated by direct counting methods, using computer programs prepared by us. The significance of association measurements [linkage disequilibrium values (ldv) estimated between all possible pairs of alleles belonging to two out of the seven loci here studied] was verified through Fisher exact tests performed on $2 \times 2$ contingency tables. All the methods here mentioned are detailed in standard text-books on statistical and population genetic methodology (Zar, 1999; Weir, 2001; Sham, 2002).

Table 1 lists the observed relative frequencies of different Y-STR alleles segregating in each of the seven loci. Table S1 (Supplementary Material) lists the observed absolute and relative frequencies of the 7-loci haplotypes among the 412 unrelated adult subjects.

Table 1 - Observed relative frequencies of Y-STR alleles segregating at each of the seven loci here reported.

\begin{tabular}{|c|c|c|c|c|c|c|c|}
\hline Allele & DYS19 & DYS389I & DYS389II & DYS390 & DYS391 & DYS392 & DYS39: \\
\hline 8 & & & & & & 0.0024 & \\
\hline 9 & & & & & 0.0558 & & \\
\hline 10 & & 0.0024 & & & 0.5073 & 0.0097 & \\
\hline 11 & & & & & 0.4053 & 0.3835 & 0.0073 \\
\hline 12 & 0.0121 & 0.1772 & & & 0.0316 & 0.0437 & 0.1893 \\
\hline 13 & 0.1117 & 0.6432 & & & & 0.5073 & 0.6626 \\
\hline $13 / 14$ & & 0.0024 & & & & & \\
\hline 14 & 0.5655 & 0.1699 & & & & 0.0461 & 0.1165 \\
\hline 15 & 0.2354 & 0.0049 & & & & 0.0024 & 0.0243 \\
\hline 16 & 0.0607 & & & & & 0.0049 & \\
\hline 17 & 0.0146 & & & & & & \\
\hline \multicolumn{8}{|l|}{18} \\
\hline \multicolumn{8}{|l|}{19} \\
\hline \multicolumn{8}{|l|}{20} \\
\hline 21 & & & & 0.0704 & & & \\
\hline 22 & & & & 0.0777 & & & \\
\hline 23 & & & & 0.2524 & & & \\
\hline 24 & & & & 0.4733 & & & \\
\hline
\end{tabular}


Table 1 (cont.)

\begin{tabular}{|c|c|c|c|c|c|c|c|}
\hline Allele & DYS19 & DYS389I & DYS389II & DYS390 & DYS391 & DYS392 & DYS393 \\
\hline 25 & & & & 0.1189 & & & \\
\hline 26 & & & 0.0049 & 0.0049 & & & \\
\hline 27 & & & 0.0121 & 0.0024 & & & \\
\hline 28 & & & 0.1408 & & & & \\
\hline 29 & & & 0.4223 & & & & \\
\hline $29 / 30$ & & & 0.0049 & & & & \\
\hline 30 & & & 0.3010 & & & & \\
\hline 31 & & & 0.0947 & & & & \\
\hline 32 & & & 0.0170 & & & & \\
\hline 33 & & & 0.0024 & & & & \\
\hline
\end{tabular}

A total of 264 different haplotypes were identified, 210 of which were unique. The most frequent haplotype (DYS19 14/DYS389I 13/DYS389II 29/DYS390 24/DYS391 11/DYS392 13/DYS393 13) was found in 31 instances $(7.52 \%)$. The second most frequent haplotype $(14 / 13 / 29 / 24 / 10 / 13 / 13)$, which differed from the previous haplotype by only a single DYS391 repeat, was shared by 14 individuals (3.39\%).

Table 2 lists the estimates of the diversity index obtained for each of the 7 Y-STR loci, the average figure calculated for these 7 loci and for the set of 7-loci haplotypes, together with their corresponding standard errors and approximate $95 \%$ confidence intervals. While diversity indices for isolated loci ranged from 0.51 to 0.70 with an overall average value of 0.60 , the 7 -loci haplotype diversity index was of the order of 0.99 , as expected, since $210 / 264$ $(79.5 \%)$ of all haplotypes were unique, each occurring with a frequency of about 0.002 .

Table 3 lists the results of association tests performed between the genes of possible pairs of Y-STR loci. Since the number of different statistical tests performed was 859 , the critical alpha rejection level (for testing the null hypoth-

Table 2 - Estimates of Nei's diversity index (di), with corresponding standard errors [se(di)] and approximate $95 \%$ confidence intervals for each individual locus, for their average value, and for the complete 7-loci haplotypes.

\begin{tabular}{lccc}
\hline & di & se(di) & $95 \%$ c.i.(di) \\
\hline locus 393 & 0.5109 & 0.1550 & $0.2071-0.8148$ \\
locus 19 & 0.6083 & 0.1184 & $0.3762-0.8404$ \\
locus 391 & 0.5743 & 0.0897 & $0.3985-0.7501$ \\
locus 389I & 0.5260 & 0.1315 & $0.2682-0.7837$ \\
locus 389II & 0.7018 & 0.0620 & $0.5803-0.8233$ \\
locus 390 & 0.6871 & 0.0874 & $0.5159-0.8583$ \\
locus 392 & 0.5914 & 0.0711 & $0.4521-0.7307$ \\
average & 0.6000 & 0.1068 & $0.3905-0.8094$ \\
all 7 loci & 0.9883 & 0.0017 & $0.9850-0.9916$ \\
\hline
\end{tabular}

esis of $\mathrm{ldv}=0$ ) was adjusted following Bonferroni's method, giving a corrected alpha critical value of 0.00006 . Therefore, Table 3 lists only the pairs of linked Y-STR loci (out of the 859 tested for linkage disequilibrium) with linkage disequilibrium values $[\mathrm{D}(\mathrm{i}, \mathrm{j})]$ significantly different from zero at the level of $6 \times 10^{-5}$ or less. As expected, many (if not most) of these very significant values occurred preferentially between pairs of contiguous loci.

In forensic cases, in which multiple male aggressors are involved, the autosomal STR profiles often provide inconclusive results. Y-STR markers are being increasingly used as potential tools for distinguishing low levels of male DNA in the presence of excess female DNA, which occurs in many sexual assault samples. Due to the haploid nature of Y-STRs, in cases where multiple males are contributors, the number of donors can be estimated by the presence of additional alleles in a $\mathrm{Y}$ chromosome profile, usually being interpreted as an admixture of more than one contributor. The most commonly used Y-STR markers are single-copy loci, with the exception of the DYS385 locus. However, many regions of the $\mathrm{Y}$-chromosome are duplicated or even triplicated in some individuals and this fact can thus complicate potential mixture interpretation (Kayser et al., 2000; Bosch and Jobling, 2003; Çakir et al., 2004; Kurihara et al., 2004; Butler et al., 2005; Diederiche et al., 2005;). The precise estimation of the frequency of duplicated mutated Y-STR alleles is thus very important in forensic genetic analyses, because the presence of multiple peaks can be misinterpreted as mixed profiles (Diederiche et al., 2005). In the present study, one sample had a 7 Y-STR haplotype with double peaks at the DYS389I locus, and another presented a 7 Y-STR haplotype with double peaks at DYS389I and DYS389II. The analysis of these two samples was increased to 16 markers, using the AmpF/STR Yfiler Kit, and a double peak was also found in the locus DYS439 of the second sample. Each one of the two pairs of father and son had the same haplotype (Table S2).

The set of 7 Y-STR loci was typed in 222 father-son pairs. Upon 1,554 allele transmissions, six de novo muta- 
Table 3 - Results of association tests and estimates of linkage disequilibrium values between possible pairs of Y-STR loci. Only pairs of loci for which the hypothesis of no association was discarded with a probability value less than 0.00006 are shown (see text).

\begin{tabular}{|c|c|c|c|c|c|c|}
\hline \multirow[t]{2}{*}{ loci } & \multicolumn{2}{|c|}{ hap } & \multirow{2}{*}{$\begin{array}{c}\text { observ. } \\
\text { freq. }\end{array}$} & \multirow{2}{*}{$\begin{array}{l}\text { expect. } \\
\text { freq. }\end{array}$} & \multirow[t]{2}{*}{$D(i, j)$} & \multirow[t]{2}{*}{$\mathrm{p}$ (Fisher) } \\
\hline & $\mathrm{i}$ & $\mathrm{j}$ & & & & \\
\hline 393(i) vs. 391(j) & 13 & 11 & 0.3228 & 0.2686 & 0.0542 & $<0.000005$ \\
\hline \multirow[t]{4}{*}{ 393(i) vs. 392(j) } & 12 & 11 & 0.1189 & 0.0726 & 0.0463 & $<0.000005$ \\
\hline & 12 & 13 & 0.0534 & 0.0960 & -0.0426 & 0.00001 \\
\hline & 13 & 11 & 0.1820 & 0.2541 & -0.0721 & $<0.000005$ \\
\hline & 13 & 13 & 0.4199 & 0.3361 & 0.0838 & $<0.000005$ \\
\hline 19(i) vs. 391(j) & 14 & 11 & 0.2791 & 0.2292 & 0.0499 & 0.00003 \\
\hline \multirow[t]{2}{*}{ 19(i) vs. 390(j) } & 14 & 21 & 0.0049 & 0.0398 & -0.0350 & $<0.000005$ \\
\hline & 15 & 21 & 0.0437 & 0.0166 & 0.0271 & $<0.000005$ \\
\hline \multirow[t]{3}{*}{ 19(i) vs. 392(j) } & 14 & 11 & 0.1214 & 0.2169 & -0.0955 & $<0.000005$ \\
\hline & 14 & 13 & 0.3908 & 0.2869 & 0.1039 & $<0.000005$ \\
\hline & 16 & 13 & 0.0024 & 0.0308 & -0.0284 & $<0.000005$ \\
\hline \multirow[t]{6}{*}{ 391(i) vs. 389I(j) } & 10 & 12 & 0.1383 & 0.0899 & 0.0485 & $<0.000005$ \\
\hline & 11 & 12 & 0.0316 & 0.0718 & -0.0403 & 0.00001 \\
\hline & 10 & 12 & 0.0704 & 0.0394 & 0.0310 & $<0.000005$ \\
\hline & 10 & 1314 & 0.1699 & 0.2401 & -0.0702 & $<0.000005$ \\
\hline & 11 & 12 & 0.0049 & 0.0315 & -0.0266 & 0.00002 \\
\hline & 11 & 1314 & 0.2597 & 0.1918 & 0.0679 & $<0.000005$ \\
\hline \multirow[t]{6}{*}{ 391(i) vs. 392(j) } & 9 & 11 & 0.0510 & 0.0214 & 0.0296 & $<0.000005$ \\
\hline & 9 & 13 & 0.0049 & 0.0283 & -0.0235 & 0.00002 \\
\hline & 10 & 11 & 0.2767 & 0.1945 & 0.0822 & $<0.000005$ \\
\hline & 10 & 13 & 0.1723 & 0.2573 & -0.0850 & $<0.000005$ \\
\hline & 11 & 11 & 0.0534 & 0.1554 & -0.1020 & $<0.000005$ \\
\hline & 11 & 13 & 0.3010 & 0.2056 & 0.0953 & $<0.000005$ \\
\hline \multirow[t]{7}{*}{ 389I(i) vs. 389II(j) } & 12 & 28 & 0.1044 & 0.0249 & 0.0794 & $<0.000005$ \\
\hline & 12 & 30 & 0.0097 & 0.0533 & -0.0436 & $<0.000005$ \\
\hline & 13 & 28 & 0.0364 & 0.0905 & -0.0541 & $<0.000005$ \\
\hline & 13 & 29 & 0.3544 & 0.2716 & 0.0827 & $<0.000005$ \\
\hline & 14 & 28 & 0.0000 & 0.0239 & -0.0239 & 0.00001 \\
\hline & 14 & 29 & 0.0170 & 0.0718 & -0.0548 & $<0.000005$ \\
\hline & 15 & 30 & 0.1044 & 0.0511 & 0.0532 & $<0.000005$ \\
\hline \multirow[t]{3}{*}{ 389I(i) vs. 390(j) } & 12 & 22 & 0.0413 & 0.0138 & 0.0275 & $<0.000005$ \\
\hline & 12 & 24 & 0.0364 & 0.0839 & -0.0475 & $<0.000005$ \\
\hline & 13 & 24 & 0.3544 & 0.3044 & 0.0499 & 0.00002 \\
\hline \multirow[t]{2}{*}{ 389I(i) vs. 392(j) } & 12 & 11 & 0.1141 & 0.0679 & 0.0461 & $<0.000005$ \\
\hline & 12 & 13 & 0.0413 & 0.0899 & -0.0486 & $<0.000005$ \\
\hline \multirow[t]{2}{*}{ 389II(i) vs. 392(j) } & 29 & 11 & 0.1019 & 0.1620 & -0.0600 & $<0.000005$ \\
\hline & 29 & 13 & 0.2718 & 0.2142 & 0.0576 & $<0.000005$ \\
\hline \multirow[t]{7}{*}{ 390(i) vs. 392(j) } & 21 & 11 & 0.0631 & 0.0270 & 0.0361 & $<0.000005$ \\
\hline & 21 & 13 & 0.0024 & 0.0357 & -0.0333 & $<0.000005$ \\
\hline & 22 & 11 & 0.0680 & 0.0298 & 0.0382 & $<0.000005$ \\
\hline & 22 & 13 & 0.0073 & 0.0394 & -0.0321 & $<0.000005$ \\
\hline & 24 & 11 & 0.0947 & 0.1815 & -0.0868 & $<0.000005$ \\
\hline & 24 & 13 & 0.3228 & 0.2401 & 0.0827 & $<0.000005$ \\
\hline & 24 & 14 & 0.0437 & 0.0218 & 0.0219 & 0.00001 \\
\hline
\end{tabular}


Table 4 - Estimated mutation rates for Y-STR individual loci, together with an overall estimate (average 7-loci figure) and the corresponding $95 \%$ binomial exact confidence intervals for each estimate.

\begin{tabular}{lcccc}
\hline System & $\begin{array}{c}\text { Allelic } \\
\text { transfers }\end{array}$ & Mutations & $\begin{array}{c}\text { Mutation } \\
\text { rate }\left(\times 10^{-3}\right)\end{array}$ & $\begin{array}{c}95 \% \text { C.I. } \\
\left(\times 10^{-3}\right)\end{array}$ \\
\hline DYS19 & 222 & 0 & 0 & \\
DYS389 I & 222 & 0 & 0 & \\
DYS389 II & 222 & 0 & 0 & \\
DYS390 & 222 & 2 & 9.01 & $0.00-32.15$ \\
DYS391 & 222 & 3 & 13.51 & $2.83-38.97$ \\
DYS392 & 222 & 1 & 4.50 & $0.04-24.82$ \\
DYS393 & 222 & 0 & 0 & \\
\hline Total & 1554 & 6 & 3.86 & $1.49-8.34$ \\
\hline
\end{tabular}

tions were observed, one mutation had occurred at the DYS392 locus (14 to 13), two mutations at the DYS390 locus (24 to $23 ; 22$ to 24$)$, and three mutations took place at the DYS391 locus (12 to $10 ; 12$ to $11 ; 11$ to 10 ) (Table 4). Except for two cases (DYS390 and DYS391), all were single-step mutations, and only a single mutation occurred during each father-son transmission.

\section{Acknowledgments}

This work was partially supported by FAPESP and LIM-HC-FMUSP, Brazil. IPATIMUP is partially supported by Fundação para a Ciência e a Tecnologia, through POCI (Programa Operacional Ciência e Inovação 2010).

\section{References}

Betz A, Babler G, Dietl G, Steil X, Weyermann G and Pflug W (2001) DYS STR analysis with epithelial cells in a rape case. Forensic Sci Int 118:126-130.

Bosch E and Jobling MA (2003) Duplications of the AZFa region of the human Y chromosome are mediated by homologous recombination between HERVs and are compatible with male fertility. Hum Mol Genet 12:341-34.

Butler JM, Decker AE, Kline MC and Vallone PM (2005) Chromosomal duplications along the Y-chromosome and their potential impact on Y-STR interpretation. J Forensic Sci 50:1-7.

Cainé L, Corte-Real F, Vieira DN, Carvalho M, Serra A, Lopes V and Vide MC (2005) Allele frequencies and haplotypes of 8 Y-chromosomal STRs in the Santa Catarina population of southern Brazil. Forensic Sci Int 148:75-79.

Çakir AH, Celebioglu A and Yardimci E (2004) Y-STR haplotypes in Central Anatolia region of Turkey. Forensic Sci Int 144:59-64.

Carvalho-Silva DR, Tarazona-Santos E, Rocha J, Pena SDJ and Santos FR (2006) Y chromosome diversity in Brazilians: Switching perspectives from slow to fast evolving markers. Genetica 126:251-260.

Cerri N, Ricci U, Sani I, Verzeletti A and de Ferrari F (2003) Mixed stains from sexual assault cases: Autosomal or
Y-Chromosome short tandem repeat? Croat Med J 44:289292.

Costa NA, Silva B and Moura-Neto RS (2002) Y-chromosome variation in a Rio de Janeiro, Brazil, population sample. Forensic Sci Int 126:254-257.

Delfin FC, Madrid BJ, Tan MP and Ungria MCA (2005) Y-STR analysis for detection and objective confirmation of child sexual abuse. Int J Legal Med 119:158-163.

Diederiche M, Martín P, Amorim A, Corte-Real F and Gusmão L (2005) A case of double alleles at three Y-STR loci: Forensic implications. Int J Legal Med 119:223-225.

Domingues PM, Gusmão L, da Silva DA, Amorim A, Pereira RW and de Carvalho EF (2007) Sub Saharan Africa descendents in Rio de Janeiro (Brazil): Population and mutational data for 12 Y-STR loci. Int J Legal Med 121:238-241.

Grattapaglia D, Kalupniek S, Guimarães CS, Ribeiro MA, Diener PS and Soares CN (2005) Y-chromosome STR haplotype diversity in Brazilian populations. Forensic Sci Int 149:99107.

Gusmão L, Butler JM, Carracedo A, Gill P, Kayser M, Mayr WR, Morling N, Prinz M, Roewer L and Tyler-Smith C (2005) DNA Commission of the International Society of Forensic Genetics (ISFG): An update of the recommendations on the use of Y-STRs in forensic analysis. Int J Legal Med 26:1-10.

Johnson CL, Giles RC, Warren JH, Floyd JI and Staub RW (2005) Analysis of non-suspect samples lacking visually identifiable sperm using a Y-STR 10- Plex. J Forensic Sci 50:1116-1118.

Kayser M, Cagliá A, Corach D, Fretwell N, Gehrig C, Graziosi G, Heidorn F, Herrmann S, Herzog B, Hidding M, et al. (1997) Evaluation of Y-chromosomal STRs: A multicenter study. Int J Legal Med 110:125-133.

Kayser M, Roewer L, Hedman M, Henke L, Henke J, Brauer S, Kruger C, Krawczak M, Nagy M, Dobosz T, et al. (2000) Characteristics and frequency of germline mutations at microsatellite loci from the human Y chromosome, as revealed by direct observation in father/son pairs. Am J Hum Genet 66:1580-1588.

Kurihara R, Yamamoto T, Uchihi R, Li SL, Yoshimoto T, Ohtaki H, Kamiyama K and Katsumata Y (2004) Mutations in 14 Y-STR loci among Japanese father-son haplotypes. Int J Legal Med 118:125-131.

Miller SA, Dykes DD and Polesky HF (1988) A simple salting-out procedure for extracting DNA from human nucleated cells. Nucleic Acids Res 6:1215.

Palha TJBF, Rodrigues EMR and Santos SEB (2007) Y-chromosomal STR haplotypes in a population from the Amazon region, Brazil. Forensic Sci Int 166:233-239.

Sham P (2002) Statistics in Human Genetics. Arnold, London, $290 \mathrm{pp}$.

Shewale JG, Nasir H, Schneida E, Gross AM, Budowle B and Sinha SK (2004) Y-Chromosome STR system, Y-PlexTM 12, for forensic casework: Development and validation. J Forensic Sci 49:1278-1290.

Shewale JG, Sikka SC, Schneida E and Sinha SK (2003) DNA profiling of azoospermic semen samples from vasectomized males by using Y-Plex TM 6 amplification Kit. J Forensic Sci 48:127-129.

Sibille I, Duverneuil C, Grandmaison GL, Guerrouache K, Teissière F, Durigon M and de Mazancourt P (2002) Y-STR DNA amplification as biological evidence in sexually as- 
saulted female victims with no cytological detection of spermatozoa. Forensic Sci Int 125:212-216.

Silva DA, Carvalho E, Costa G, Tavares L, Amorim A and Gusmão L (2006) Y-chromosome genetic variation in Rio de Janeiro population. Am J Hum Biol 18:829-837.

Weir BS (2001) Genetic Data Analysis II. Sinauer, Sunderland, $445 \mathrm{pp}$.

Zar JH (1999) Biostatistical Analysis. Prentice Hall, Upper Saddle River, $663 \mathrm{pp}$.

\section{Internet Resources}

Instituto Brasileiro de Geografia e Estatística (IBGE), www.ibge. gov.br (2007).

\section{Supplementary Material}

The following online material is available for this article:

- Table S1. Observed absolute and relative frequencies of STR-Y 7-loci haplotypes.

- Table S2. Y-STR haplotype profiles showing the presence of additional alleles.

This material is available as part of the online article from http://www.scielo.br.gmb. Associate Editor: Francisco Mauro Salzano

License information: This is an open-access article distributed under the terms of the Creative Commons Attribution License, which permits unrestricted use, distribution, and reproduction in any medium, provided the original work is properly cited. 
TABLE S1 - Observed absolute and relative frequencies of STR-Y 7-loci haplotypes.

\begin{tabular}{|c|c|c|c|c|c|c|c|c|c|}
\hline $\mathrm{H}$ & 19 & 3891 & $389 I I$ & 390 & 391 & 392 & 393 & obs.no. & Freq. \\
\hline 1 & 12 & 12 & 28 & 22 & 10 & 11 & 13 & 1 & 0.00243 \\
\hline 2 & 12 & 12 & 28 & 23 & 11 & 16 & 11 & 1 & 0.00243 \\
\hline 3 & 12 & 13 & $29 / 30$ & 24 & 11 & 13 & 13 & 1 & 0.00243 \\
\hline 4 & 12 & 13 & 31 & 24 & 10 & 11 & 13 & 1 & 0.00243 \\
\hline 5 & 12 & 14 & 30 & 25 & 11 & 13 & 13 & 1 & 0.00243 \\
\hline 6 & 13 & 12 & 28 & 23 & 10 & 11 & 13 & 1 & 0.00243 \\
\hline 7 & 13 & 12 & 28 & 24 & 10 & 14 & 12 & 1 & 0.00243 \\
\hline 8 & 13 & 12 & 29 & 24 & 10 & 11 & 13 & 1 & 0.00243 \\
\hline 9 & 13 & 12 & 30 & 24 & 10 & 14 & 13 & 1 & 0.00243 \\
\hline 10 & 13 & 13 & 28 & 23 & 11 & 13 & 13 & 1 & 0.00243 \\
\hline 11 & 13 & 13 & 28 & 24 & 11 & 13 & 13 & 1 & 0.00243 \\
\hline 12 & 13 & 13 & 29 & 23 & 11 & 13 & 13 & 1 & 0.00243 \\
\hline 13 & 13 & 13 & 29 & 24 & 9 & 11 & 13 & 2 & 0.00485 \\
\hline 14 & 13 & 13 & 29 & 24 & 10 & 11 & 12 & 1 & 0.00243 \\
\hline 15 & 13 & 13 & 29 & 24 & 10 & 13 & 13 & 2 & 0.00485 \\
\hline 16 & 13 & 13 & 29 & 24 & 11 & 13 & 12 & 1 & 0.00243 \\
\hline 17 & 13 & 13 & 29 & 24 & 11 & 13 & 13 & 2 & 0.00485 \\
\hline 18 & 13 & 13 & 29 & 24 & 12 & 13 & 12 & 1 & 0.00243 \\
\hline 19 & 13 & 13 & 29 & 25 & 10 & 13 & 13 & 1 & 0.00243 \\
\hline 20 & 13 & 13 & 29 & 25 & 11 & 13 & 13 & 1 & 0.00243 \\
\hline 21 & 13 & 13 & 30 & 23 & 10 & 11 & 13 & 3 & 0.00728 \\
\hline 22 & 13 & 13 & 30 & 23 & 10 & 11 & 14 & 1 & 0.00243 \\
\hline 23 & 13 & 13 & 30 & 23 & 10 & 11 & 12 & 1 & 0.00243 \\
\hline 24 & 13 & 13 & 30 & 23 & 10 & 11 & 13 & 5 & 0.01214 \\
\hline 25 & 13 & 13 & 30 & 23 & 10 & 11 & 14 & 1 & 0.00243 \\
\hline 26 & 13 & 13 & 30 & 25 & 9 & 11 & 14 & 1 & 0.00243 \\
\hline 27 & 13 & 13 & 30 & 25 & 10 & 11 & 13 & 1 & 0.00243 \\
\hline 28 & 13 & 13 & 31 & 23 & 10 & 11 & 13 & 1 & 0.00243 \\
\hline 29 & 13 & 13 & 31 & 23 & 10 & 15 & 13 & 1 & 0.00243 \\
\hline 30 & 13 & 13 & 31 & 24 & 10 & 11 & 12 & 1 & 0.00243 \\
\hline 31 & 13 & 13 & 31 & 24 & 10 & 11 & 13 & 1 & 0.00243 \\
\hline 32 & 13 & 13 & 31 & 24 & 10 & 14 & 13 & 1 & 0.00243 \\
\hline 33 & 13 & 14 & 30 & 23 & 10 & 13 & 13 & 1 & 0.00243 \\
\hline 34 & 13 & 14 & 30 & 24 & 9 & 11 & 13 & 5 & 0.01214 \\
\hline 35 & 13 & 14 & 30 & 24 & 9 & 11 & 14 & 1 & 0.00243 \\
\hline 36 & 13 & 14 & 30 & 24 & 10 & 14 & 13 & 1 & 0.00243 \\
\hline 37 & 13 & 14 & 30 & 25 & 10 & 11 & 13 & 1 & 0.00243 \\
\hline 38 & 13 & 14 & 33 & 23 & 10 & 11 & 12 & 1 & 0.00243 \\
\hline 39 & 14 & 12 & 27 & 23 & 10 & 13 & 13 & 1 & 0.00243 \\
\hline 40 & 14 & 12 & 27 & 24 & 10 & 14 & 12 & 1 & 0.00243 \\
\hline 41 & 14 & 12 & 27 & 24 & 11 & 13 & 13 & 1 & 0.00243 \\
\hline 42 & 14 & 12 & 27 & 25 & 10 & 13 & 14 & 1 & 0.00243 \\
\hline 43 & 14 & 12 & 28 & 22 & 10 & 11 & 13 & 1 & 0.00243 \\
\hline 44 & 14 & 12 & 28 & 22 & 10 & 11 & 15 & 1 & 0.00243 \\
\hline 45 & 14 & 12 & 28 & 23 & 9 & 13 & 13 & 1 & 0.00243 \\
\hline 46 & 14 & 12 & 28 & 23 & 10 & 11 & 12 & 2 & 0.00485 \\
\hline 47 & 14 & 12 & 28 & 23 & 10 & 11 & 13 & 5 & 0.01214 \\
\hline 48 & 14 & 12 & 28 & 23 & 10 & 13 & 13 & 1 & 0.00243 \\
\hline 49 & 14 & 12 & 28 & 23 & 11 & 11 & 13 & 1 & 0.00243 \\
\hline 50 & 14 & 12 & 28 & 23 & 11 & 12 & 13 & 1 & 0.00243 \\
\hline 51 & 14 & 12 & 28 & 23 & 12 & 13 & 13 & 1 & 0.00243 \\
\hline 52 & 14 & 12 & 28 & 24 & 0 & 13 & 13 & 2 & 0.0 \\
\hline
\end{tabular}


TABLE S1 (Continued)

\begin{tabular}{|c|c|c|c|c|c|c|c|c|c|}
\hline $\mathrm{H}$ & 19 & 3891 & 38911 & 390 & 391 & 392 & 393 & obs.no. & Freq. \\
\hline 53 & 14 & 12 & 28 & 24 & 11 & 13 & 13 & 2 & 0.00485 \\
\hline 54 & 14 & 12 & 28 & 24 & 11 & 14 & 13 & 1 & 0.00243 \\
\hline 55 & 14 & 12 & 28 & 24 & 12 & 13 & 13 & 1 & 0.00243 \\
\hline 56 & 14 & 12 & 28 & 27 & 10 & 13 & 13 & 1 & 0.00243 \\
\hline 57 & 14 & 12 & 29 & 22 & 10 & 11 & 12 & 1 & 0.00243 \\
\hline 58 & 14 & 12 & 29 & 22 & 10 & 11 & 14 & 1 & 0.00243 \\
\hline 59 & 14 & 12 & 29 & 23 & 10 & 11 & 12 & 1 & 0.00243 \\
\hline 60 & 14 & 12 & 29 & 25 & 10 & 13 & 13 & 1 & 0.00243 \\
\hline 61 & 14 & 12 & 29 & 25 & 11 & 12 & 13 & 1 & 0.00243 \\
\hline 62 & 14 & 12 & 30 & 22 & 10 & 11 & 13 & 1 & 0.00243 \\
\hline 63 & 14 & 12 & 30 & 23 & 10 & 12 & 14 & 1 & 0.00243 \\
\hline 64 & 14 & 13 & 26 & 23 & 10 & 13 & 13 & 1 & 0.00243 \\
\hline 65 & 14 & 13 & 28 & 23 & 11 & 13 & 13 & 1 & 0.00243 \\
\hline 66 & 14 & 13 & 28 & 24 & 10 & 13 & 13 & 1 & 0.00243 \\
\hline 67 & 14 & 13 & 28 & 24 & 11 & 13 & 13 & 4 & 0.00971 \\
\hline 68 & 14 & 13 & 28 & 24 & 11 & 13 & 14 & 1 & 0.00243 \\
\hline 69 & 14 & 13 & 28 & 24 & 11 & 14 & 12 & 1 & 0.00243 \\
\hline 70 & 14 & 13 & 29 & 21 & 11 & 13 & 14 & 1 & 0.00243 \\
\hline 71 & 14 & 13 & 29 & 22 & 9 & 11 & 12 & 1 & 0.00243 \\
\hline 72 & 14 & 13 & 29 & 22 & 10 & 11 & 12 & 1 & 0.00243 \\
\hline 73 & 14 & 13 & 29 & 22 & 10 & 11 & 13 & 1 & 0.00243 \\
\hline 74 & 14 & 13 & 29 & 23 & 10 & 11 & 12 & 3 & 0.00728 \\
\hline 75 & 14 & 13 & 29 & 23 & 10 & 13 & 13 & 2 & 0.00485 \\
\hline 76 & 14 & 13 & 29 & 23 & 11 & 11 & 12 & 3 & 0.00728 \\
\hline 77 & 14 & 13 & 29 & 23 & 11 & 11 & 13 & 1 & 0.00243 \\
\hline 78 & 14 & 13 & 29 & 23 & 11 & 12 & 13 & 1 & 0.00243 \\
\hline 79 & 14 & 13 & 29 & 23 & 11 & 13 & 13 & 4 & 0.00971 \\
\hline 80 & 14 & 13 & 29 & 24 & 9 & 13 & 13 & 1 & 0.00243 \\
\hline 81 & 14 & 13 & 29 & 24 & 10 & 11 & 12 & 1 & 0.00243 \\
\hline 82 & 14 & 13 & 29 & 24 & 10 & 13 & 12 & 7 & 0.01699 \\
\hline 83 & 14 & 13 & 29 & 24 & 10 & 13 & 13 & 14 & 0.03398 \\
\hline 84 & 14 & 13 & 29 & 24 & 10 & 14 & 13 & 2 & 0.00485 \\
\hline 85 & 14 & 13 & 29 & 24 & 11 & 12 & 13 & 1 & 0.00243 \\
\hline 86 & 14 & 13 & 29 & 24 & 11 & 13 & 12 & 3 & 0.00728 \\
\hline 87 & 14 & 13 & 29 & 24 & 11 & 13 & 13 & 31 & 0.07524 \\
\hline 88 & 14 & 13 & 29 & 24 & 11 & 13 & 14 & 2 & 0.00485 \\
\hline 89 & 14 & 13 & 29 & 24 & 11 & 14 & 12 & 1 & 0.00243 \\
\hline 90 & 14 & 13 & 29 & 24 & 11 & 14 & 13 & 1 & 0.00243 \\
\hline 91 & 14 & 13 & 29 & 24 & 12 & 13 & 13 & 1 & 0.00243 \\
\hline 92 & 14 & 13 & 29 & 25 & 10 & 13 & 13 & 3 & 0.00728 \\
\hline 93 & 14 & 13 & 29 & 25 & 11 & 11 & 12 & 1 & 0.00243 \\
\hline 94 & 14 & 13 & 29 & 25 & 11 & 13 & 11 & 1 & 0.00243 \\
\hline 95 & 14 & 13 & 29 & 25 & 11 & 13 & 13 & 6 & 0.01456 \\
\hline 96 & 14 & 13 & 29 & 25 & 12 & 13 & 13 & 1 & 0.00243 \\
\hline 97 & 14 & 13 & 29 & 26 & 10 & 11 & 12 & 1 & 0.00243 \\
\hline 98 & 14 & 13 & 29 & 26 & 11 & 13 & 12 & 1 & 0.00243 \\
\hline 99 & 14 & 13 & 30 & 21 & 10 & 11 & 13 & 1 & 0.00243 \\
\hline 100 & 14 & 13 & 30 & 22 & 10 & 11 & 13 & 1 & 0.00243 \\
\hline 101 & 14 & 13 & 30 & 22 & 10 & 11 & 14 & 1 & 0.00243 \\
\hline 102 & 14 & 13 & 30 & 22 & 10 & 13 & 13 & 1 & 0.00243 \\
\hline 103 & 14 & 13 & 30 & 23 & 10 & 11 & 11 & 1 & 0.00243 \\
\hline 104 & 14 & 13 & 30 & 23 & 10 & 11 & 12 & 5 & 0.01214 \\
\hline 105 & 14 & 13 & 30 & 23 & 10 & 13 & 13 & 3 & 0.00728 \\
\hline 106 & 14 & 13 & 30 & 23 & 11 & 11 & 12 & 1 & 0.00243 \\
\hline
\end{tabular}


TABLE S1 (Continued)

\begin{tabular}{|c|c|c|c|c|c|c|c|c|c|}
\hline $\mathrm{H}$ & 19 & 3891 & $389 I 1$ & 390 & 391 & 392 & 393 & obs.no. & Freq. \\
\hline 107 & 14 & 13 & 30 & 23 & 11 & 13 & 13 & 1 & 0.00243 \\
\hline 108 & 14 & 13 & 30 & 24 & 10 & 10 & 13 & 1 & 0.00243 \\
\hline 109 & 14 & 13 & 30 & 24 & 10 & 11 & 12 & 2 & 0.00485 \\
\hline 110 & 14 & 13 & 30 & 24 & 10 & 13 & 12 & 1 & 0.00243 \\
\hline 111 & 14 & 13 & 30 & 24 & 10 & 13 & 13 & 2 & 0.00485 \\
\hline 112 & 14 & 13 & 30 & 24 & 10 & 13 & 14 & 1 & 0.00243 \\
\hline 113 & 14 & 13 & 30 & 24 & 11 & 12 & 13 & 1 & 0.00243 \\
\hline 114 & 14 & 13 & 30 & 24 & 11 & 13 & 12 & 1 & 0.00243 \\
\hline 115 & 14 & 13 & 30 & 24 & 11 & 13 & 13 & 7 & 0.01699 \\
\hline 116 & 14 & 13 & 30 & 24 & 11 & 13 & 14 & 1 & 0.00243 \\
\hline 117 & 14 & 13 & 30 & 24 & 12 & 13 & 13 & 2 & 0.00485 \\
\hline 118 & 14 & 13 & 30 & 25 & 10 & 13 & 13 & 1 & 0.00243 \\
\hline 119 & 14 & 13 & 30 & 25 & 10 & 16 & 13 & 1 & 0.00243 \\
\hline 120 & 14 & 13 & 30 & 25 & 11 & 13 & 13 & 3 & 0.00728 \\
\hline 121 & 14 & 13 & 31 & 23 & 10 & 11 & 12 & 1 & 0.00243 \\
\hline 122 & 14 & 13 & 31 & 23 & 10 & 13 & 13 & 2 & 0.00485 \\
\hline 123 & 14 & 13 & 31 & 24 & 9 & 11 & 13 & 1 & 0.00243 \\
\hline 124 & 14 & 13 & 31 & 24 & 11 & 13 & 13 & 1 & 0.00243 \\
\hline 125 & 14 & 14 & 29 & 23 & 10 & 10 & 14 & 1 & 0.00243 \\
\hline 126 & 14 & 14 & 29 & 23 & 10 & 13 & 12 & 1 & 0.00243 \\
\hline 127 & 14 & 14 & 29 & 23 & 10 & 13 & 13 & 1 & 0.00243 \\
\hline 128 & 14 & 14 & 29 & 23 & 11 & 13 & 13 & 1 & 0.00243 \\
\hline 129 & 14 & 14 & 29 & 24 & 11 & 14 & 12 & 2 & 0.00485 \\
\hline 130 & 14 & 14 & 30 & 22 & 10 & 13 & 13 & 1 & 0.00243 \\
\hline 131 & 14 & 14 & 30 & 23 & 9 & 11 & 13 & 1 & 0.00243 \\
\hline 132 & 14 & 14 & 30 & 23 & 10 & 11 & 12 & 1 & 0.00243 \\
\hline 133 & 14 & 14 & 30 & 23 & 10 & 13 & 13 & 1 & 0.00243 \\
\hline 134 & 14 & 14 & 30 & 23 & 11 & 13 & 12 & 1 & 0.00243 \\
\hline 135 & 14 & 14 & 30 & 23 & 11 & 13 & 13 & 2 & 0.00485 \\
\hline 136 & 14 & 14 & 30 & 23 & 12 & 13 & 13 & 1 & 0.00243 \\
\hline 137 & 14 & 14 & 30 & 24 & 10 & 13 & 12 & 1 & 0.00243 \\
\hline 138 & 14 & 14 & 30 & 24 & 10 & 13 & 13 & 2 & 0.00485 \\
\hline 139 & 14 & 14 & 30 & 24 & 11 & 13 & 12 & 1 & 0.00243 \\
\hline 140 & 14 & 14 & 30 & 24 & 11 & 13 & 13 & 7 & 0.01699 \\
\hline 141 & 14 & 14 & 30 & 24 & 11 & 13 & 14 & 2 & 0.00485 \\
\hline 142 & 14 & 14 & 30 & 24 & 11 & 14 & 13 & 2 & 0.00485 \\
\hline 143 & 14 & 14 & 30 & 24 & 12 & 13 & 13 & 1 & 0.00243 \\
\hline 144 & 14 & 14 & 30 & 24 & 12 & 13 & 14 & 1 & 0.00243 \\
\hline 145 & 14 & 14 & 30 & 25 & 10 & 11 & 12 & 1 & 0.00243 \\
\hline 146 & 14 & 14 & 30 & 25 & 10 & 13 & 12 & 1 & 0.00243 \\
\hline 147 & 14 & 14 & 30 & 25 & 10 & 13 & 13 & 2 & 0.00485 \\
\hline 148 & 14 & 14 & 30 & 25 & 11 & 12 & 13 & 1 & 0.00243 \\
\hline 149 & 14 & 14 & 30 & 25 & 11 & 13 & 13 & 1 & 0.00243 \\
\hline 150 & 14 & 14 & 31 & 23 & 9 & 11 & 12 & 1 & 0.00243 \\
\hline 151 & 14 & 14 & 31 & 23 & 9 & 11 & 14 & 1 & 0.00243 \\
\hline 152 & 14 & 14 & 31 & 23 & 10 & 11 & 13 & 1 & 0.00243 \\
\hline 153 & 14 & 14 & 31 & 23 & 10 & 13 & 13 & 1 & 0.00243 \\
\hline 154 & 14 & 14 & 31 & 24 & 11 & 11 & 13 & 1 & 0.00243 \\
\hline 155 & 14 & 14 & 31 & 24 & 11 & 13 & 13 & 2 & 0.00485 \\
\hline 156 & 14 & 14 & 31 & 25 & 11 & 11 & 13 & 1 & 0.00243 \\
\hline 157 & 14 & 14 & 31 & 25 & 11 & 13 & 13 & 1 & 0.00243 \\
\hline 158 & 14 & 14 & 32 & 23 & 10 & 11 & 13 & 1 & 0.00243 \\
\hline 159 & 14 & 14 & 32 & 24 & 11 & 13 & 13 & 1 & 0.00243 \\
\hline 160 & 14 & 14 & 32 & 25 & 10 & 12 & 14 & 1 & 0.00243 \\
\hline
\end{tabular}


TABLE S1 (Continued)

\begin{tabular}{|c|c|c|c|c|c|c|c|c|c|}
\hline $\mathrm{H}$ & 19 & 3891 & 389II & 390 & 391 & 392 & 393 & obs.no. & Freq. \\
\hline 161 & 14 & 15 & 31 & 25 & 11 & 13 & 13 & 1 & 0.00243 \\
\hline 162 & 14 & 15 & 32 & 23 & 10 & 13 & 13 & 1 & 0.00243 \\
\hline 163 & 15 & 10 & 29 & 21 & 10 & 10 & 13 & 1 & 0.00243 \\
\hline 164 & 15 & 12 & 26 & 21 & 10 & 11 & 13 & 1 & 0.00243 \\
\hline 165 & 15 & 12 & 27 & 23 & 10 & 13 & 13 & 1 & 0.00243 \\
\hline 166 & 15 & 12 & 28 & 21 & 10 & 11 & 14 & 1 & 0.00243 \\
\hline 167 & 15 & 12 & 28 & 22 & 10 & 11 & 13 & 1 & 0.00243 \\
\hline 168 & 15 & 12 & 28 & 22 & 10 & 11 & 14 & 2 & 0.00485 \\
\hline 169 & 15 & 12 & 28 & 23 & 10 & 11 & 12 & 1 & 0.00243 \\
\hline 170 & 15 & 12 & 28 & 23 & 10 & 11 & 14 & 1 & 0.00243 \\
\hline 171 & 15 & 12 & 28 & 24 & 10 & 11 & 12 & 1 & 0.00243 \\
\hline 172 & 15 & 12 & 28 & 24 & 10 & 11 & 13 & 2 & 0.00485 \\
\hline 173 & 15 & 12 & 28 & 24 & 11 & 11 & 12 & 1 & 0.00243 \\
\hline 174 & 15 & 12 & 28 & 25 & 10 & 11 & 12 & 1 & 0.00243 \\
\hline 175 & 15 & 12 & 28 & 25 & 11 & 13 & 12 & 1 & 0.00243 \\
\hline 176 & 15 & 12 & 28 & 25 & 11 & 13 & 13 & 1 & 0.00243 \\
\hline 177 & 15 & 12 & 29 & 21 & 10 & 11 & 14 & 1 & 0.00243 \\
\hline 178 & 15 & 12 & 29 & 22 & 10 & 11 & 12 & 1 & 0.00243 \\
\hline 179 & 15 & 12 & 29 & 22 & 10 & 11 & 14 & 2 & 0.00485 \\
\hline 180 & 15 & 12 & 29 & 23 & 10 & 11 & 12 & 2 & 0.00485 \\
\hline 181 & 15 & 12 & 29 & 23 & 10 & 11 & 13 & 1 & 0.00243 \\
\hline 182 & 15 & 12 & 29 & 23 & 10 & 11 & 15 & 1 & 0.00243 \\
\hline 183 & 15 & 12 & 29 & 23 & 10 & 12 & 14 & 1 & 0.00243 \\
\hline 184 & 15 & 12 & 29 & 23 & 10 & 13 & 13 & 1 & 0.00243 \\
\hline 185 & 15 & 12 & 30 & 22 & 10 & 11 & 14 & 1 & 0.00243 \\
\hline 186 & 15 & 13 & 28 & 22 & 11 & 11 & 13 & 1 & 0.00243 \\
\hline 187 & 15 & 13 & 28 & 23 & 10 & 11 & 13 & 1 & 0.00243 \\
\hline 188 & 15 & 13 & 28 & 25 & 10 & 13 & 13 & 1 & 0.00243 \\
\hline 189 & 15 & 13 & 28 & 25 & 11 & 13 & 13 & 1 & 0.00243 \\
\hline 190 & 15 & 13 & 29 & 21 & 10 & 11 & 14 & 1 & 0.00243 \\
\hline 191 & 15 & 13 & 29 & 22 & 10 & 11 & 13 & 1 & 0.00243 \\
\hline 192 & 15 & 13 & 29 & 22 & 11 & 13 & 12 & 1 & 0.00243 \\
\hline 193 & 15 & 13 & 29 & 23 & 9 & 11 & 12 & 3 & 0.00728 \\
\hline 194 & 15 & 13 & 29 & 23 & 10 & 11 & 12 & 1 & 0.00243 \\
\hline 195 & 15 & 13 & 29 & 23 & 10 & 11 & 13 & 1 & 0.00243 \\
\hline 196 & 15 & 13 & 29 & 23 & 10 & 11 & 14 & 1 & 0.00243 \\
\hline 197 & 15 & 13 & 29 & 23 & 10 & 12 & 14 & 1 & 0.00243 \\
\hline 198 & 15 & 13 & 29 & 23 & 10 & 13 & 13 & 2 & 0.00485 \\
\hline 199 & 15 & 13 & 29 & 23 & 10 & 12 & 12 & 1 & 0.00243 \\
\hline 200 & 15 & 13 & 29 & 23 & 10 & 13 & 13 & 3 & 0.00728 \\
\hline 201 & 15 & 13 & 29 & 23 & 11 & 13 & 13 & 9 & 0.02184 \\
\hline 202 & 15 & 13 & 29 & 23 & 11 & 14 & 13 & 2 & 0.00485 \\
\hline 203 & 15 & 13 & 29 & 23 & 12 & 13 & 14 & 1 & 0.00243 \\
\hline 204 & 15 & 13 & 29 & 25 & 10 & 13 & 13 & 1 & 0.00243 \\
\hline 205 & 15 & 13 & 29 & 25 & 11 & 13 & 13 & 1 & 0.00243 \\
\hline 206 & 15 & 13 & 29 & 25 & 12 & 13 & 13 & 1 & 0.00243 \\
\hline 207 & 15 & 13 & 30 & 21 & 9 & 11 & 14 & 1 & 0.00243 \\
\hline 208 & 15 & 13 & 30 & 21 & 10 & 11 & 13 & 1 & 0.00243 \\
\hline 209 & 15 & 13 & 30 & 21 & 10 & 11 & 14 & 1 & 0.00243 \\
\hline 210 & 15 & 13 & 30 & 21 & 10 & 11 & 15 & 1 & 0.00243 \\
\hline 211 & 15 & 13 & 30 & 21 & 11 & 11 & 13 & 1 & 0.00243 \\
\hline 212 & 15 & 13 & 30 & 21 & 11 & 11 & 14 & 1 & 0.00243 \\
\hline 213 & 15 & 13 & 30 & 21 & 12 & 11 & 13 & 1 & 0.00243 \\
\hline 214 & 15 & 13 & 30 & 22 & 10 & 11 & 12 & 1 & 0.00243 \\
\hline
\end{tabular}


TABLE S1 (Continued)

\begin{tabular}{|c|c|c|c|c|c|c|c|c|c|}
\hline $\mathbf{H}$ & 19 & 3891 & 38911 & 390 & 391 & 392 & 393 & obs.no. & Freq. \\
\hline 215 & 15 & 13 & 30 & 23 & 9 & 11 & 12 & 2 & 0.00485 \\
\hline 216 & 15 & 13 & 30 & 23 & 11 & 13 & 13 & 1 & 0.00243 \\
\hline 217 & 15 & 13 & 30 & 24 & 10 & 11 & 13 & 1 & 0.00243 \\
\hline 218 & 15 & 13 & 30 & 24 & 10 & 12 & 15 & 1 & 0.00243 \\
\hline 219 & 15 & 13 & 30 & 24 & 11 & 13 & 13 & 1 & 0.00243 \\
\hline 220 & 15 & 13 & 30 & 24 & 11 & 13 & 14 & 2 & 0.00485 \\
\hline 221 & 15 & 13 & 30 & 24 & 11 & 14 & 13 & 1 & 0.00243 \\
\hline 222 & 15 & 13 & 31 & 21 & 10 & 10 & 13 & 1 & 0.00243 \\
\hline 223 & 15 & 13 & 31 & 21 & 10 & 11 & 13 & 3 & 0.00728 \\
\hline 224 & 15 & 13 & 31 & 21 & 10 & 11 & 15 & 1 & 0.00243 \\
\hline 225 & 15 & 13 & 31 & 21 & 11 & 11 & 13 & 1 & 0.00243 \\
\hline 226 & 15 & 13 & 31 & 24 & 10 & 11 & 12 & 1 & 0.00243 \\
\hline 227 & 15 & 13 & 31 & 24 & 10 & 11 & 13 & 1 & 0.00243 \\
\hline 228 & 15 & 13 & 31 & 24 & 11 & 13 & 13 & 1 & 0.00243 \\
\hline 229 & 15 & 13 & 31 & 25 & 10 & 11 & 13 & 1 & 0.00243 \\
\hline 230 & 15 & 13 & 32 & 22 & 10 & 11 & 14 & 1 & 0.00243 \\
\hline 231 & 15 & 14 & 29 & 24 & 11 & 13 & 13 & 1 & 0.00243 \\
\hline 232 & 15 & 14 & 30 & 24 & 11 & 13 & 13 & 1 & 0.00243 \\
\hline 233 & 15 & 14 & 31 & 22 & 10 & 11 & 14 & 1 & 0.00243 \\
\hline 234 & 15 & 14 & 31 & 23 & 10 & 12 & 14 & 1 & 0.00243 \\
\hline 235 & 15 & 14 & 31 & 23 & 10 & 12 & 15 & 1 & 0.00243 \\
\hline 236 & 15 & 14 & 32 & 24 & 10 & 11 & 12 & 1 & 0.00243 \\
\hline 237 & 15 & 14 & 32 & 24 & 11 & 13 & 13 & 1 & 0.00243 \\
\hline 238 & 16 & 12 & 28 & 21 & 10 & 11 & 13 & 1 & 0.00243 \\
\hline 239 & 16 & 12 & 28 & 22 & 10 & 11 & 13 & 3 & 0.00728 \\
\hline 240 & 16 & 12 & 28 & 22 & 10 & 11 & 14 & 1 & 0.00243 \\
\hline 241 & 16 & 12 & 29 & 21 & 10 & 11 & 15 & 1 & 0.00243 \\
\hline 242 & 16 & 12 & 29 & 21 & 11 & 11 & 13 & 2 & 0.00485 \\
\hline 243 & 16 & 12 & 29 & 25 & 10 & 11 & 13 & 1 & 0.00243 \\
\hline 244 & 16 & 13 & 29 & 22 & 10 & 8 & 15 & 1 & 0.00243 \\
\hline 245 & 16 & 13 & 29 & 23 & 9 & 11 & 12 & 1 & 0.00243 \\
\hline 246 & 16 & 13 & 29 & 23 & 10 & 12 & 14 & 1 & 0.00243 \\
\hline 247 & 16 & 13 & 29 & 23 & 11 & 11 & 12 & 1 & 0.00243 \\
\hline 248 & 16 & 13 & 29 & 23 & 11 & 12 & 14 & 1 & 0.00243 \\
\hline 249 & 16 & 13 & 29 & 25 & 11 & 12 & 13 & 1 & 0.00243 \\
\hline 250 & 16 & 13 & 30 & 21 & 10 & 11 & 15 & 1 & 0.00243 \\
\hline 251 & 16 & 13 & 30 & 23 & 11 & 12 & 13 & 1 & 0.00243 \\
\hline 252 & 16 & 13 & 30 & 24 & 10 & 11 & 13 & 1 & 0.00243 \\
\hline 253 & 16 & 13 & 30 & 24 & 10 & 11 & 14 & 1 & 0.00243 \\
\hline 254 & 16 & 13 & 30 & 24 & 11 & 11 & 13 & 1 & 0.00243 \\
\hline 255 & 16 & 13 & 30 & 25 & 11 & 11 & 13 & 1 & 0.00243 \\
\hline 256 & 16 & 13 & 31 & 23 & 10 & 14 & 14 & 1 & 0.00243 \\
\hline 257 & 16 & 13 & 31 & 24 & 11 & 11 & 13 & 2 & 0.00485 \\
\hline 258 & 16 & $13 / 14$ & $29 / 30$ & 25 & 11 & 13 & 13 & 1 & 0.00243 \\
\hline 259 & 17 & 13 & 28 & 22 & 10 & 11 & 13 & 1 & 0.00243 \\
\hline 260 & 17 & 13 & 30 & 21 & 10 & 11 & 14 & 1 & 0.00243 \\
\hline 261 & 17 & 13 & 30 & 24 & 11 & 11 & 13 & 1 & 0.00243 \\
\hline 262 & 17 & 14 & 30 & 21 & 10 & 11 & 15 & 1 & 0.00243 \\
\hline 263 & 17 & 14 & 31 & 21 & 10 & 11 & 13 & 1 & 0.00243 \\
\hline 264 & 17 & 14 & 31 & 21 & 10 & 11 & 14 & 1 & 0.00243 \\
\hline
\end{tabular}


TABLE S2 - Y-STR haplotype profiles showing the presence of additional alleles at DYS389II (case 1) and DYS389I, DYS389II and DYS439 (case 2), after amplification with the AmpF/STR Yfiler kit.

\begin{tabular}{|c|c|c|c|c|c|c|c|c|c|c|c|c|c|c|c|c|}
\hline Samples & DYS456 & DYS389I & DYS390 & DYS389II & DYS458 & DYS19 & DYS385 & DYS393 & DYS391 & DYS439 & DYS635 & DYS392 & GATA H4 & DYS437 & DYS438 & DYS448 \\
\hline $\begin{array}{l}\text { Case } 1 \\
\text { father }\end{array}$ & 15 & 13 & 24 & $29-30$ & 16 & 12 & $12-14$ & 13 & 11 & 11 & 23 & 13 & 21 & 15 & 12 & 20 \\
\hline Case 1 son & 15 & 13 & 24 & $29-30$ & 16 & 12 & $12-14$ & 13 & 11 & 11 & 23 & 13 & 21 & 15 & 12 & 20 \\
\hline $\begin{array}{l}\text { Case } 2 \\
\text { father }\end{array}$ & 15 & 13-14 & 25 & $29-30$ & 17 & 16 & $13-14$ & 13 & 11 & $10-13$ & 23 & 13 & 21 & 15 & 12 & 19 \\
\hline Case 2 son & 15 & 13-14 & 25 & $29-30$ & 17 & 16 & $13-14$ & 13 & 11 & $10-13$ & 23 & 13 & 21 & 15 & 12 & 19 \\
\hline
\end{tabular}

